\title{
The Study of Selection of Air Material Storehouse based on Second Generation of Genetic Algorithm in Site
}

\author{
Jinzhong Zheng ${ }^{\mathrm{a}}$, Kaitian $\mathrm{Xu}{ }^{\mathrm{a}}$, Yu Xin ${ }^{\mathrm{a}}$, Yang Cui ${ }^{\mathrm{a}}$ \\ Department of Air Material Management, Air Force Logistics College, Jiangsu 221000, China \\ a452970162@qq.com
}

Keywords: Air Material Storehouse, Site selection, Genetic algorithm, the Lagrange slack variables.

\begin{abstract}
The article advances the method of a modified genetic algorithm which applies the Lagrange slack variables to solve the sub problem about air material storehouse of permanent position distributes requirement of the army. Finally the article uses an example to verify the validity of the model and method.
\end{abstract}

\section{Air Material warehouse location model}

Logistics center location model are nonlinear mixing $0-1^{[2]}$, for which plans to build a warehouse for air material to protect the area within a known demand logistics support troops on the model make the following assumptions: The number of aircraft materials warehouse needs more than the number of troops that $n>m$; An Air Materiel warehouse can serve multiple needs of the troops, but one needs only logistical support troops by an aviation materials warehouse; The capacity of each logistics center not be less than the demand of its safeguards troops; the sum of air Materiel warehouse construction scale and security objects related demand, ensure the protection of objects to meet the demand, and an increase of $10 \%$ of the total as a contingency reserve. Based on the above assumptions, the second location model of military logistics for:

$$
\begin{aligned}
& \min \quad E=\sum_{i=1}^{m} \sum_{j=1}^{n} d_{i j} W_{j} Z_{i j}+\xi \sum_{i=1}^{m} \sum_{j=1}^{n} W_{j} Z_{i j}+\omega(1+10 \%) \sum_{i=1}^{m} \sum_{j=1}^{n} W_{j} Z_{i j} \\
& d_{i j}=\sqrt{\left(x_{i}-u_{j}\right)^{2}-\left(y_{i}-v_{j}\right)^{2}},(i=1,2, \ldots m ; j=1,2, \ldots n) \\
& \sum_{j=1}^{n} W_{j} Z_{i j} \leq a_{i} \\
& X_{S} \leq x_{i}, u_{j} \leq X_{E},(i=1,2, \ldots m ; j=1,2, \ldots n) \\
& Y_{S} \leq y_{i}, v_{j} \leq Y_{E},(i=1,2, \ldots m ; j=1,2, \ldots n) \\
& \sum_{i=1}^{m} Z_{i j}=1,(j=1,2, \ldots n) \\
& W_{j} \geq 0, \xi \geq 0, \omega \geq 0,(j=1,2, \ldots n)
\end{aligned}
$$

Where: $E$ - Air Materiel Depot security costs; $m$ - within the protection area is planned to build the number of aircraft materials warehouse; the number of troops in the $n$ - safeguard regional needs; $W_{j}$ - the first Air Force needs a timber demand; $d_{i j}$ - th material supply $i$ air material $j$ warehouse; $\left(X_{S}, Y_{S}\right)$ - th Air Materiel Depot Euclidean distance to th needs of the troops; - to protect the area starting position; $\left(X_{E}, Y_{E}\right)$ - protection area end position; $\left(x_{i}, y_{i}\right)$ - TBD location th Air Materiel warehouse; $\left(u_{j}, v_{j}\right)$ - location th needs of the troops $j$; $\xi$ - management cost ratio; $\omega$ - construction cost ratio; $Z_{i j}$ - discrete decision variables, if the $i$-th Air Materiel Depot $j$-th demand protection force, then $Z_{i j}=1$, otherwise, $Z_{i j}=0$. 


\section{Lagrangian relaxation combined with variable genetic algorithm in the warehouse location problem}

Determining a coding mode. Location Air Materiel warehouse belonging continuous variables, using a float to be encoded, chromosome length of the $2 \times m$-site; and $Z_{i j}$ can only take two values, 0 or 1 , encoded binary chromosome length of the $n \times m$-site, and ensure that each $m$ bit encoding and only one to one. In summary, a schematic model for encoding the Table 1:

Table 1 model coded to indicate the table

\begin{tabular}{c|c|c|c|c}
\hline$i_{\text {-th Air Materiel Depot }}$ & 1 & 2 & $\cdots$ & $m$ \\
\hline Location TBD $\left(x_{i}, y_{i}\right)$ & $\left(x_{1}, y_{1}\right)$ & $\left(x_{2}, y_{2}\right)$ & $\cdots$ & $\left(x_{m}, y_{m}\right)$ \\
\hline$j$-th demand forces & 1 & 2 & $\cdots$ & $n$ \\
\hline Decision variables $Z_{i j}$ & $100 \cdots 0$ & $Z_{12} Z_{22} \cdots Z_{m 2}$ & $\cdots$ & $Z_{1 n} Z_{2 n} \cdots Z_{m n}$ \\
\hline
\end{tabular}

Initial population. Randomly generate a mixed-length coding chromosome $(2 \times m+n \times m)$, constitutes the initial population. Air Materiel warehouse pending positions $\left(x_{i}, y_{i}\right), x_{i}$ randomly generated must meet in $\left[X_{S}, X_{E}\right], y_{i}$ randomly generated in $\left[Y_{S}, Y_{E}\right] ; Z_{i j}$ decision variables and the random coding. $M$ Denotes the size of the population included in the number of individuals of the population, the appropriate population size can increase the diversity of the population, and to accelerate the speed optimization algorithm can be selected according to the actual situation in between.

Fitness function. Fitness function is to evaluate the pros and cons of each chromosome group functions. The objective function of the model is to take the minimum, not directly as a fitness function, you need to construct a new fitness function, such as the formula (9):

$$
\text { Fitness }=\frac{1}{E}=\frac{1}{\sum_{i=1}^{m} \sum_{j=1}^{n} d_{i j} W_{j} Z_{i j}+\xi \sum_{i=1}^{m} \sum_{j=1}^{n} W_{j} Z_{i j}+\omega(1+10 \%) \sum_{i=1}^{m} \sum_{j=1}^{n} W_{j} Z_{i j}}
$$

Varangian relaxation variables. Fitness function and the corresponding demand of the logistics center is closely related to the distribution of forces, and thus calculate the fitness of the need to address the needs of the sub-allocation problems. Lagrange relaxation variables to solve slack variables to solve for a fixed position of logistics distribution center needs child asked ${ }^{[3]}$. Provided Lagrangian multiplier operator $\theta_{\imath}$, so that slack capacity constraint is: $\sum_{j=1}^{n} W_{j} Z_{i j} \leq a_{i}$, after conversion of the objective function is:

$$
\min E=\sum_{i=1}^{m} \sum_{j=1}^{n} d_{i j} W_{j} Z_{i j}+\xi \sum_{i=1}^{m} \sum_{j=1}^{n} W_{j} Z_{i j}+\omega(1+10 \%) \sum_{i=1}^{m} \sum_{j=1}^{n} W_{j} Z_{i j}+\sum_{i=1}^{m} \theta_{i}\left(a_{i}-\sum_{j=1}^{n} W_{j} Z_{i j}\right)
$$

Genetic Operators operating

Select operation. In this paper, roulette selection method to select the operation, so you can ensure the convergence of the genetic algorithm. $F_{k}$ Value for the adaptation of chromosome $k$, its selection probability according to equation (9) Calculated:

$$
P_{k}=\frac{F_{k}}{\sum_{k=1}^{M} F_{k}} \quad(k=1,2, \ldots M)
$$

Cross operation. This model is used to solve a single point crossover method, respectively, Air Materiel warehouse location $\left(x_{i}, y_{i}\right)$ and decision variables $Z_{i j}$ cross operation. Under the premise of satisfying the constraints, the two parts respectively encoding random point as the cross point, then 
the last two parts corresponding to the coding information code intersections between two chromosomes exchange obtain progeny chromosomes.

Mutation in the genetic algorithm according to the rules of common variants, 0 - 1 generates a first random number $\beta$, if it is less than a given sufficiently small real number $\alpha$, i.e. $\beta<\alpha$, the randomly selected genes of the chromosome location, the information code adapted to the new value. Also, make sure the chromosome mutated meets the constraints. Determination algorithm termination condition. In this paper, a fixed maximum iteration algebra algorithm termination conditions and optimum output groups of individuals as the final result.

\section{Examples of application}

Suppose in the direction of regional operations have location data and aviation materials demand 30 air material needs of the troops, as shown in Table 2. It is required to determine the three best aviation materials warehouse (A, B, C) in the protection area within the site, to provide material support for the needs of troops.

Table 2 demand troops statistics

\begin{tabular}{ccccccccc}
\hline$j$ & $\left(u_{j}, v_{j}\right)$ & $W_{j}$ & $j$ & $\left(u_{j}, v_{j}\right)$ & $W_{j}$ & $j$ & $\left(u_{j}, v_{j}\right)$ & $W_{j}$ \\
\hline 1 & $(9,91)$ & 35 & 11 & $(25,95)$ & 31 & 21 & $(20,66)$ & 34 \\
2 & $(26,31)$ & 47 & 12 & $(76,93)$ & 48 & 22 & $(56,43)$ & 38 \\
3 & $(78,28)$ & 38 & 13 & $(85,76)$ & 30 & 23 & $(92,59)$ & 47 \\
4 & $(8,12)$ & 36 & 14 & $(75,56)$ & 42 & 24 & $(79,9)$ & 42 \\
5 & $(39,42)$ & 44 & 15 & $(63,26)$ & 35 & 25 & $(89,87)$ & 39 \\
6 & $(11,26)$ & 37 & 16 & $(42,23)$ & 41 & 26 & $(54,91)$ & 44 \\
7 & $(10,64)$ & 32 & 17 & $(37,87)$ & 52 & 27 & $(62,67)$ & 48 \\
8 & $(89,40)$ & 46 & 18 & $(48,63)$ & 33 & 28 & $(31,54)$ & 36 \\
9 & $(8,47)$ & 34 & 19 & $(32,75)$ & 42 & 29 & $(18,45)$ & 30 \\
10 & $(22,17)$ & 39 & 20 & $(18,82)$ & 40 & 30 & $(67,85)$ & 41 \\
\hline
\end{tabular}

Parameter settings are: the number of the population is 20, the maximum evolution algebra 200, crossover probability is 0.6 , mutation probability is 0.1 , the management cost was 3 , the construction cost was 5, in particular the work site, according to actual adjusted appropriately. Location model and solving method based on the first two sections are constructed using the following algorithm program run Matlab2011 environment. Thus, the use of aircraft materials warehouse location model, so that security costs have been minimal air material warehouse location coordinates and distribution relationship and demand forces, as shown in Table 3.

Table 3 Air Materiel warehouse location model for solving the initial results

\begin{tabular}{cccc}
\hline Costs & \multicolumn{3}{c}{37054} \\
\hline Air Materiel Depot & Location coordinates & Demand for troops & Supply \\
\hline \multirow{2}{*}{ A } & $(37.8885$, & $1,7,11,17,19,20$, & 390 \\
& $72.4861)$ & $21,26,28,30$ & \\
B & $(21.9410$, & $2,4,5,6,9$, & 310 \\
& $23.8523)$ & $10,16,29$ & 470 \\
\hline
\end{tabular}


Using the model established and proposed the corresponding algorithm; the program has stabilized operating results, found the global optimal solution or the approximate global optimal solution. Security costs by minimizing the identified location results A- (37.8885, 72.4861), B(21.9410, 23.8523), C- (72.6610, 50.3034).

\section{Summary}

Lagrange slack variables, the calculation of complex fitness function are transformed into independent sub-problems, which simplifies the calculations. Effective solution when put military warehouse systems analysis and design in sitting questions about aviation materials warehouse and optimize its logistics system so that it can operate effectively and bring benefits to the protection needs of the troops, has a certain practicality.

\section{References}

[1] Jianrong Feng. Global Hybrid Optimization Algorithm for Solving MINLP Problems. JOURNAL OF SYSTEM SIMULATION [J], 2011, 17 (8): p.1859—1863

[2] Jian Wu, Zhongke Shi. Selection of Distribution Center's Location Based on Genetic Algorithm. JOURNAL OF SOUTH CHINA UNIVERSITY OF TECHNOLOGY (NATURAL SCIENCE EDITION) [J], 2010, 32(6): p.72-74

[3] Yi Tao, Jianqing Zhu, Ming Li. Optimization of locations of logistics centers based on improved Genetic Algorithm. COMPUTER ENGINEERING AND APPLICATIONS [J], 2011, 43(25): p.221-223. 